\title{
Antihypertensive Drug Utilization in Two Districts of Northern Cyprus
}

\author{
Mevhibe Tamirci*, Rümeysa Demirdamar \\ European University of Lefke, Faculty of Pharmacy \\ Lefke, Northern Cyprus, TR-10 Mersin, Turkey
}

\begin{abstract}
Aim. Since the irrational use of medicine increases the risk of morbidity and mortality in hypertension, this study was aimed to evaluate antihypertensive pharmacotherapy at two districts of Northern Cyprus (NC).

Material and methods. A total of 148 prescriptions including antihypertensive drugs ( $n=181$ ) were obtained by the aid of five pharmacists between November 2017 - August 2018 and evaluated regarding the antihypertensive drug choices and good prescribing principles. The prescribed antihypertensive agents were classified according to the Anatomical Therapeutic Chemical Classification System (ATC) 2, 3 and 5 and the prescriptions were evaluated regarding the availability of format information.

Results. The mean of antihypertensive agents per prescription was 1.2 \pm 0.6 . According to the ATC-3 subclassification, beta-blockers (22.1\%), angiotensin receptor blockers (ARBs) $(22.1 \%)$, calcium channel blockers (20.4\%), angiotensin converting enzyme inhibitor (ACE) inhibitors (17.7\%), diuretics (13.8\%) and anti-adrenergic drugs (3.9\%) were prescribed. According to the ATC-5 subclassification, the most common prescribed antihypertensives were metoprolol (16.6\%), amlodipine (16.0\%), furosemide (8.8\%), captopril $(7.7 \%)$ and losartan (6.6\%). There were no significant relation between the prescribed antihypertensive agents and gender and the physicians serving either in governmental or private hospitals. There were shortcomings in the prescriptions such as age, diagnosis and drug information which were crucial for the chosen of an appropriate antihypertensive agent.

Conclusion. This first pharmacoepidemiological study about antihypertensive drug utilization in NC indicates the imperfections of physicians in terms of prescribing antihypertensive agents according to the guidelines and writing a "legible and good" prescription that contains full information. These findings underline necessity of educational interventions for physicians to disseminate rational use of medicine (RUM) in the NC.
\end{abstract}

Keywords: rational use of medicine, physicians, prescribing patterns, pharmacoepidemiology.

For citation: Tamirci M., Demirdamar R. Antihypertensive Drug Utilization in Two Districts of Northern Cyprus. Rational Pharmacotherapy in Cardiology 2019;15(4):467-477. DOI:10.20996/1819-6446-2019-15-4-467-477

\section{Применение антигипертензивных препаратов на примере двух областей Северного Кипра}

Мевхибе Тамирджи*, Рюмейса Демирдамар

Европейский университет Лефке

Турция, Северный Кипр, Лефке, ТР-10 Мерсин

Цель. Поскольку нерациональное применение лекарственных средств при гипертонии приводит к росту заболеваемости и смертности, целью данного исследования является оценка антигипертензивной фармакотерапии на основе данных из двух районов Северного Кипра. Материал и методы. В период с ноября 2017 г. по август 2018 г. при помощи 5-ти фармацевтов было собрано 148 рецептов, содержащих назначение антигипертензивных препаратов (общее количество 181), проведена оценка рациональности выбора препаратов и надлежащего алгоритма их назначения. Назначенные антигипертензивные действующие вещества определялись по системе анатомо-терапевтическо-химической классификации (АТХ) как класс 2, 3 и 5. Рецепты оценивались с точки зрения соответствия формату.

Результаты. Среднее количество антигипертензивных препаратов в рецепте составило 1,2士0,6. В рамках подкласса АТХ-3 выделялись бета-блокаторы (22,1\%), блокаторы рецепторов ангиотензина (БРА) (22,1\%), антагонисты кальция (20,4\%), ингибиторы ангиотензинпревращающего фермента (АПФ) (17,7\%), диуретики (13,8\%) и анти-адренергические средства (3,9\%). По подклассу АТХ-5 часто назначаемыми гипотензивными веществами являлись метопролол $(16,6 \%)$, амлодипин $(16,0 \%)$, фуросемид $(8,8 \%)$, каптоприл (7,7\%) и лозартан (6,6\%). Статистически значимой корреляции типа антигипертензивного вещества и пола врача, его назначившего, а также его принадлежности государственному или частному лечебному учреждению не выявлено. В исследуемых рецептах обнаружены недочеты оформления, такие как отсутствие сведений о возрасте пациента, его диагнозе, а также указаний по применению препарата. Эта информация необходима для корректного выбора антигипертензивного препарата.

Заключение. Это первое фармакоэпидемиологическое исследование применения гипотензивных препаратов на территории Северного Кипра выявило недостатки в работе врачей, назначающих антигипертензивные средства, в частности, неполное следование рекомендациям и выписка рецепта, не содержащего полную информацию. Эти результаты свидетельствуют о необходимости обучающих мероприятий для врачей для внедрения принципов рационального использования лекарственных средств в регионах Северного Кипра.

Ключевые слова: рациональная фармакотерапия, врачи, схемы назначений, фармакоэпидемиология.

Для цитирования: Тамирджи М., Демирдамар Д. Применение антигипертензивных препаратов на примере двух областей Северного Кипра. Рациональная Фармакотерапия в Кардиологии 2019;15(4):467-477. DOI:10.20996/1819-6446-2019-15-4-467-477

*Corresponding Author (Автор, ответственный за переписку): mtamirci@eul.edu.tr

Received / Поступила: 29.05.2019

Accepted / Принята в печать: 02.07.2019 
Increased blood pressure is accounted as the leading risk for deaths in developed countries [1]. The mechanisms such as increases in sympathoadrenal and reninangiotensin systems, decreasing of the baroreceptor sensitivity and impairments of the ion channels are involved in the etiology of hypertension [2]. Therefore, various different drugs with different mechanism of action are found in market for hypertension treatment. Untreated hypertension can cause further seconder diseases such as myocardial infarction, stroke, congestive heart failure, renal disease and peripheral vascular disease [3]. For the successful management of hypertension, several international guidelines are available [4-7]. These guidelines serve physicians to choose rational medications in doses that meet the requirement of the patients with the lowest cost as defined by World Health Organization (WHO) [8]. However, some epidemiological studies reported the unsatisfactory results of the antihypertensive treatment due to irrational drug use $[9,10]$. Irrational use of drugs might differ from country to country and depends on physicians, pharmacists as well as patients. Therefore, the antihypertensive drug prescription patterns of the physicians, the drug dispensing performance of the pharmacists and the antihypertensive drug usage habits of the patients are important factors throughout the course of the treatment. In literature, any research on the use of antihypertensive drugs in Northern Cyprus (NC) does not exist. So, this pharmacoepidemiological research aims to evaluate the antihypertensive drug prescription patterns of the physicians and to analyses whether physicians follow the available guidelines in NC.

\section{Material and methods}

According to the last census, the permanent residents of the country population was around 300000 [11] and 200 community pharmacists were registered in the relevant professional associations, in 2017 in NC.

Data presented in this study were collected by 5 community pharmacists between November 2017 August 2018 in two districts (Lefka and Omorphu) of NC. There are 5 districts (Famagusta, Kyrenia, Nicosia, Omorphu, Lefka), Lefka and Omorphu are located in the North West and 13 pharmacist were serving in these districts in 2017.

In this study, the hypertension drug prescription patterns of the physicians who serving in these two districts were evaluated. During the 9-month study period, a total of 148 prescriptions including antihypertensive drugs were collected and analyzed. For prescription analysis, a copy of the script was received and the original was given back to the pharmacists. Prescriptions were evaluated according to the below
В развитых странах повышенное артериальное давление (АД) является лидирующим фактором риска увеличения смертности населения [1]. В формировании артериальной гипертензии (АГ) участвуют следующие механизмы: активация симпатоадреналовой и ренинангиотензиновой систем, снижение чувствительности барорецепторов и нарушения транспортных ионных каналов [2]. Вследствие этого на рынке присутствует ряд препаратов для лечения АГ с различными принципами действия. Неконтролируемая, не леченная АГ может привести к развитию таких событий как инфаркт миокарда, инсульт, застойная сердечная недостаточность, почечная недостаточность и заболевания периферических артерий [3]. Существуют несколько международных рекомендаций для успешного лечения АГ [4-7]. Эти клинические рекомендации позволяют врачам рационально выбирать препараты и дозировку в соответствии с состоянием пациента, а также снижать финансовые затраты на лечение, как требует Всемирная организация здравоохранения (ВО3) [8]. Однако некоторые эпидемиологические исследования отмечают неудовлетворительные результаты лечения АГ из-за нерационального применения медикаментов $[9,10]$. Некорректное применение препаратов отличается в разных странах и зависит от назначающих специалистов, фармацевтов и самих пациентов. Таким образом, на процесс лечения АГ влияют несколько важных факторов - схемы назначений антигипертензивных препаратов, которые используют терапевты, распределение медикаментов в аптеках и предпочтения пациентов в приеме средств для понижения давления. В существующих источниках не упоминается ни одно исследование применения антигипертензивных препаратов на территории Северного Кипра. Настоящее исследование имеет цель выявить схемы назначения антигипертензивных препаратов, которые используют специалисты, а также проанализировать, насколько врачи Северного Кипра следуют существующим клиническим руководствам.

\section{Материал и методы}

По последним данным учета населения в 2017 г. в Северном Кипре постоянно проживают около 300000 человек [1 1], на них приходится примерно 200 районных фармацевтов, зарегистрированных в профессиональных сообществах.

Данные, использованные для исследования, получены у 5-ти районных фармацевтов (регионы Северного Кипра Лефка и Морфу) за период с ноября 2017 г. по август 2018 г. В целом Северный Кипр состоит из 5 регионов (Фамагуста, Кирения, Никосия, Морфу, Лефка); Лефка и Морфу находятся на северо-западе, в 2017 г. их обслуживали 13 фармацевтов.

В настоящем исследовании анализируются данные о схемах назначения антигипертензивных препаратов 
criteria (based on "Guide to Good Prescribing" indicator):

a. Format of the script (Presence of date, name of the physician, branch of the physician, availability of the diagnosis, name and age of the patient)

b. Readable handwriting

c. Presence of generic and trade name of the drug

d. Presence of the pharmaceutical form, dosage and total amount of the drug

e. Presence of instructions and warnings

$f$. Number of drugs per prescription

g. Number of antihypertensive drugs per prescription

The prescriptions further analyzed to classify the prescribed drugs according to Anatomical Therapeutic Chemical Classification System (ATC) 1, 2, 3 and 5 and compare the usage frequency of these drugs with national guidelines.

For this study, an approval was obtained from the European University of Lefke Institutional Review Board.

The Statistical Package for the Social Sciences (SPSS, version 20.0) was used for data analyses. The frequency analyses and descriptive statistics were applied.

\section{Results}

A total of 148 prescriptions including antihypertensive drugs were collected and analyzed. The average number of drugs per prescription was $2.1 \pm 1.9$ and average number of antihypertensive drugs per prescription was $1.2 \pm 0.6$.

The prescriptions were analyzed for legibility and format. The $40.5 \%$ of the prescriptions were written by the physicians serving in governmental hospitals whereas $41.9 \%$ of them were written by physicians serving in private hospitals or clinics. It was observed that only $3.4 \%(n=5)$ of the prescriptions included "hypertension diagnosis" and only $2.0 \%$ of them $(n=3)$ included the age of the patient. When the availability of the "diagnosis" and "age information" of the patient was compared between the prescriptions written by governmental and private physicians, it was recognized that $80.0 \%(n=4)$ of the diagnosis and $100.0 \%$ of the age information was written by the governmental physicians. Most of the prescriptions included the name/surname of the patient (89.9\%) and the physician (76.4\%). Almost half of the prescriptions $(47.3 \%)$ included the date. The $41.2 \%$ of the prescriptions were written by the internal medicine specialist and $14.9 \%$ of them were written by a cardiologist.

It was recognized that most of the drug dosages and active ingredient amount written legibly (92.4\% and $82.3 \%$ of the drugs, respectively) by the physicians. The $67.5 \%$ of the legibly written drug dosages врачами из этих двух регионов. За 9 мес исследования было собрано и проанализировано 148 рецептов, в которых присутствовали гипотензивные препараты. Для анализа рецепта с него снималась копия, оригинал возвращался фармацевту. Рецепты оценивались по нижеперечисленным критериям (основаны на руководстве по надлежащему назначению лекарственных препаратов):

а. Формат рецепта (указаны дата, имени и фамилии врача, его специализация, диагноз, имя и возраст пациента)

б. Разборчивость почерка

в. Наличие наименования дженерика и торгового наименования препарата

г. Указание лекарственной формы, дозы и общего требуемого количества препарата

д. Наличие рекомендаций по применению и предостережений

е. Количество препаратов в рецепте

ж. Количество антигипертензивных препаратов в рецепте

Далее рецепты анализировались на соответствие препаратов классам 1, 2, 3 и 5 Анатомо-терапевтическохимической классификации (ATX), и частота их назначения сравнивалась с национальными руководствами.

Настоящее исследование получило одобрение экспертного совета Европейского Университета Лефке.

Для анализа данных применялась программа статистики для социальных наук (SPSS, версия 20.0). Использовался метод частотного анализа и описательная статистика. Данные представлены в виде среднего (M) и стандартного отклонения (SD).

\section{Результаты}

Всего было собрано и проанализировано 148 рецептов, в которых присутствовали антигипертензивные препараты. Среднее количество препаратов в рецепте составило 2,1 $\pm 1,9$, среднее количество антигипертензивных препаратов в рецепте - 1,2 20,6 .

Далее оценивались удобочитаемость и формат рецептов: 40,5\% рецептов были выписаны врачами государственных медицинских учреждений, в то время как $41,9 \%$ рецептов выписаны врачами частных клиник или стационаров. Только в 3,4\% случаев (5 рецептов) содержался диагноз «гипертензия», и только в 2,0\% случаев (3 рецепта) был указан возраст пациента. При сравнении наличия диагноза и возраста пациента в рецептах, выписанных врачами государственных или частных медицинских учреждений, обнаружено, что диагноз указан в $80 \%$ случаев (4 рецепта), и возраст указан в 100\% случаев врачами государственных лечебных учреждений. Большинство рецептов содержали имя/фамилию пациента $(89,9 \%)$ и врача $(76,4 \%)$. Около половины рецептов были датированы $(47,3 \%) ; 41,2 \%$ 
was "once a day" and $16.4 \%$ of them was "twice a day". On the other hand, almost half of the prescribed drugs either did not include the pharmaceutical form information or include this information illegibly (40.7\% and $4.4 \%$ of the drugs, respectively). The most common written pharmaceutical form was "tablet" (76.7\%). Most physicians did not write instructions (70.7\%) and warnings (70.3\%) for the prescribed drugs.

According to the ATC-1 classification, 65\% $(n=206)$ of the total of 317 prescribed drugs belonged to cardiovascular system drug group (" $\mathrm{C}$ "), as expected. The rest of the prescribed drugs were alimentary tract and metabolism drugs ("A", $11.7 \%$ ), respiratory system drugs ("R", 6.6\%), blood and blood forming organ drugs ("B", $6.0 \%$ ), nervous system drugs, (" $\mathrm{N}$ ", $5.4 \%)$, musculoskeletal system drugs ("M", 2.5\%), anti-infective ("J", 1.6\%) and others (1.2\%).

According to the ATC-2 subclassification, $87.9 \%$ $(n=181)$ of the prescribed cardiovascular system drugs were the antihypertensive. The most common prescribed antihypertensive drugs were the agents acting on renin-angiotensin-system ("C09", 39.8\%), betablocking agents ("C07", 22.1\%), calcium channel blockers ("C08", 20.4\%), diuretics ("C03", 14.4\%) and other antihypertensive ("CO2", 3.3\%) (Table 1).

The most common prescribed drugs in renin-angiotensin system were angiotensin converting enzyme (ACE) inhibitors ("C09A", 41.7\%), angiotensin receptor blocker (ARB), combinations ("C09D", 29.2\%) and $A R B$, plain ("CO9C", 26.4\%). It was recognized that almost all of the prescribed calcium channel blockers were selective calcium channel blockers with mainly

Table 1. The distribution of the prescribed antihypertensive drugs according to the ATC-2 subclassification (number of prescribed antihypertensives = 181)

Таблица 1. Распределение назначенных антигипертензивных препаратов по подклассу АTX-

2 (общее количество назначенных антигипертензивных препаратов - 181)

\begin{tabular}{|c|c|c|}
\hline $\begin{array}{l}\text { S. № } \\
\text { № п.п. }\end{array}$ & $\begin{array}{l}\text { Drug class } \\
\text { Класс препарата }\end{array}$ & $\mathrm{n}(\%)$ \\
\hline 1 & $\begin{array}{l}\text { Renin-Angiotensin System Drugs / Препараты, } \\
\text { влияющие на ренин-ангиотензиновую систему }\end{array}$ & $72(39.8)$ \\
\hline 2 & Beta-blockers / Бета-адреноблокаторы & $40(11.1)$ \\
\hline 3 & Calcium-channel blockers / Антагонисты кальция & $37(20.4)$ \\
\hline 4 & Diuretics / Диуретики & $26(14.4)$ \\
\hline 5 & $\begin{array}{l}\text { Anti-adrenergic drugs } \\
\text { Антиадренергические средства }\end{array}$ & $6(3.3)$ \\
\hline $\begin{array}{l}\text { ATC - anat } \\
\text { ATX - анат }\end{array}$ & $\begin{array}{l}\text { mical therapeutic chemical classification } \\
\text { мо-терапевтическо-химическая классификация }\end{array}$ & \\
\hline
\end{tabular}

рецептов выписаны врачами-терапевтами, 14,9\% врачами-кардиологами.

Нужно отметить, что в большинстве рецептов режим дозирования препарата и количество действующего вещества были написаны разборчиво (для 92,4\% и 82,3\% препаратов, соответственно). Режим дозирования в 67,5\% удобочитаемых рецептов был указан как «раз в день», в 16,4\% рецептов - «два раза в день». С другой стороны, почти для половины выписанных препаратов не была указана лекарственная форма, или указана неразборчиво (для 40,7\% и 4,4\% препаратов, соответственно). Самая часто применяемая лекарственная форма - таблетки (76,7\%). Большинство врачей не указывают рекомендации по применению (70,7\%) и предостережения (70,3\%) для назначенных лекарственных средств.

Как и ожидалось, 65\% (206 препаратов) из общего числа назначенных (317) относились к категории сердечно-сосудистых средств (группа С, класс АTX-1). Остальные назначенные препараты представляли категории: препараты, влияющие на пищеварительный тракт и обмен веществ (группа A, 11,7\%), препараты для лечения заболеваний дыхательной системы (группа R, 6,6\%), препараты влияющие на органы кроветворения и кровь (группа В, 6,0\% ), средства для лечения заболеваний нервной системы (группа N, 5,4\%), костно-мышечной системы (группа М, 2,5\%), противомикробные препараты (группа J, 1,6\%) и прочие (1,2\%).

Большинство из назначенных сердечно-сосудистых средств ( 181 препарат, 87,9\%) являлись антигипертензивными по подклассу АTX-2. Наиболее часто назначаемыми гипотензивными средствами оказались средства, влияющие на ренин-ангиотензиновую систему («С09», 39,8\%), бета-адреноблокаторы («С07», 22,1\%), антагонисты кальция («С08», 20,4\%), диуретики («С03», 14,4\% ) и прочие антигипертензивные препараты («С02», 3,3\%) (табл. 1).

Наиболее распространенными препаратами для регуляции ренин-ангиотензиновой системы стали ингибиторы ангиотензин-превращающего фермента (АПФ) ( (C09A», 41,7\% ), блокаторы рецепторов ангиотензина II (БРА) в комбинации с другими препаратами ( СC09D», 29,2\%) и непосредственно БРА ( “С09С», 26,4\%). Практически все назначенные антагонисты кальция относились к группе селективных с преимущественным влиянием на сосуды («С08С», 94,6\%). Назначенные диуретики в основном относились к группам петлевых («С03С», 64,0\%) и калийсберегающих («C03D», 32,0\%).

По подклассу ATX-3 наиболее назначаемыми препаратами являлись бета-адреноблокаторы (22,1\%), БРА $(22,1 \%)$, антагонисты кальция $(20,4 \%)$ и ингибиторы АПФ (17,7\%) (табл. 2).

По подклассу ATX-5 часто назначаемыми антигипертензивными веществами являлись метопролол 
Table 2. The distribution of the prescribed antihypertensive drugs according to the ATC-3 subclassification (number of prescribed antihypertensives = 181)

Таблица 2. Распределение назначенных антигипертензивных препаратов по подклассу ATX-3 (общее количество назначенных антигипертензивных препаратов - 181)

\begin{tabular}{|c|c|c|}
\hline $\begin{array}{l}\text { S. No } \\
\text { № п.п. }\end{array}$ & $\begin{array}{l}\text { Drug class } \\
\text { Класс препарата }\end{array}$ & $\mathrm{n}(\%)$ \\
\hline 1 & Beta-blockers / Бета-адреноблокаторы & $40(22.1)$ \\
\hline \multirow[t]{2}{*}{2} & Angiotensin Receptor blockers & \\
\hline & Блокаторы рецепторов ангиотензина & $40(22.1)$ \\
\hline 3 & Calcium Channel Blockers / Антагонисты кальция & $37(20.4)$ \\
\hline 4 & ACE-inhibitors / Ингибиторы АПФ & $32(17.7)$ \\
\hline 5 & Diuretics / Диуретики & 25(13.8) \\
\hline \multirow[t]{2}{*}{6} & Anti-adrenergic drugs & \\
\hline & Антиадренергические средства & $7(3.9)$ \\
\hline \multicolumn{3}{|c|}{$\begin{array}{l}\text { ATC - anatomical therapeutic chemical classification, } \\
\text { ACE - angiotensin converting enzyme }\end{array}$} \\
\hline \multicolumn{3}{|c|}{ AТХ - анатомо-терапевтическо-химическая классификация, } \\
\hline
\end{tabular}

vascular effects ("C08C", 94.6\%). The most common prescribed diuretics were high-ceiling diuretics ("C03C", 64.0\%) and potassium-sparing agents ("C03D", 32.0\%).

According to the ATC-3 subclassification, it was recognized that the most prescribed drugs were beta blockers (22.1\%), ARBs (22.1\%), calcium channel blockers (20.4\%) and ACE inhibitors (17.7\%) (Table 2).

According to the ATC- 5 subclassification, the most common prescribed antihypertensive were metoprolol ("C07AB02", 16.6\%), amlodipine ("C08CA01", $16.0 \%)$, furosemide ("C03CA01", 8.8\%), captopril ("CO9AA01", 7.7\%), losartan ("CO9CA01", 6.6\%), valsartan and diuretics ("C09DA03", 6.1\%).

Most of the prescriptions ( $73.0 \%, n=108)$ were monotherapy whereas $23.0 \%$ of them $(n=34)$ were dual combinations and $4.0 \%(n=6)$ of them were triple combination therapy. The most preferred dualcombination therapy was ACE inhibitors + diuretics fix combinations (Table 3 ). The $66.7 \%$ of the triple drug combinations $(n=4)$ were beta blockers + ARBs + thiazide diuretics (prescribed separately).

It was also analyzed that the choice of antihypertensive drug groups by physicians were not associated with gender and governmental/private hospital $(p>0.05)$. According to the ATC-2 subclassification, the most prescribed drug group both in governmental and private hospitals were the agents acting on reninangiotensin system (ARBs + ACE inhibitors). In addi-
(«С07АВ02», 16,6\%), амлодипин («С08СА01», 16,0\%), фуросемид («СОЗСА01», 8,8\%), каптоприл («С09AА01», 7,7\%) и лозартан («CO9DA03», 6,6\%), валсартан и диуретики («CO9DA03», 6, 1\%).

Большинство назначений были в виде монотерапии (73,0\%, 108 рецептов), но в 23,0\% случаев встречались комбинации двух препаратов (34 рецепта), в то время как комбинация трех препаратов применялась в 4,0\% случаев (6 рецептов). Наиболее предпочтительной комбинацией двух препаратов явилось сочетание ингибиторов АПФ с диуретиками в виде комбинированной лекарственной формы (табл. 3). В комбинации из трех препаратов в 66,7\% случаев (4 рецепта) участвовали бета-адреноблокаторы, БРА и тиазидные диуретики, где каждый назначался отдельно.

Анализ выбора антигипертензивных средств врачами не выявил его корреляцию с полом специалиста и принадлежностью государственному или частному лечебному заведению $(p>0,05)$. По подклассу АTX-2 наиболее часто назначаемыми препаратами были вещества, влияющие на ренин-ангиотензиновую систему (БРА + ингибиторы АПФ), как среди врачей государственных медицинских учреждений, так и частных. Дополнительно по подклассу ATX-3 предпочитаемыми группами препаратов явились бета-блокаторы, БРА и ингибиторы АПФ (табл. 4).

По подклассу ATX-2 также выявлено, что специалисты обоих полов наиболее часто назначают вещества, влияющие на ренин-ангиотензиновую систему (БРА + ингибиторы АПФ). Однако врачи женского пола предпочитают назначать ингибиторы АПФ, БРА и бета-блокаторы, а врачи-мужчины - антагонисты кальция и бета-адреноблокаторы. БРА выписывались как препарат второй линии (табл. 5).

\section{Обсуждение}

Целью нашего исследования был анализ схем назначения антигипертензивных препаратов на основе оценки выписанных рецептов, в которых встречались данные препараты. Судя по данным, содержащимся в литературе, это первое фармакоэпидемиологическое исследование рационального использования антигипертензивных лекарственных средств врачами Северного Кипра. Выявленные недочеты в схемах назначения свидетельствуют о необходимости проведения обучения врачей для улучшения их тактики назначений.

В настоящем исследовании среднее количество препаратов в рецепте $(2,1)$ соответствует данным исследования, проведенного в Бахрейне $(2,12)$, и несколько ниже, чем показатель исследования, проведенного в Стамбуле, Турция $(3,8)[12,13]$. Полипрагмазия является одной из проблем при рациональном подходе к назначению лекарственных средств, особенно, у пожилых пациентов. Полипрагмазия нежелательна, так как может 
Table 3. The distribution of the dual drug combinations in prescriptions (the total number of prescriptions $=148$, total number of prescriptions with dual combination $=34$ )

Таблица 3. Распределение комбинаций двух препаратов в рецептах (общее количество рецептов - 148, общее количество комбинаций двух препаратов - 34)

\begin{tabular}{|c|c|c|}
\hline $\begin{array}{l}\text { S. № } \\
\text { № п.п. }\end{array}$ & $\begin{array}{l}\text { Drug class } \\
\text { Класс препарата }\end{array}$ & $\mathrm{n}(\%)$ \\
\hline 1 & Angiotensin receptor blockers + diuretics (fixed combination) / БРА+диуретики (комбинированный препарат) & $18(52.9)$ \\
\hline 2 & Loop diuretics + potassiumspairing diuretics (prescribed separately) / Петлевые диуретики+калий сберегающие диуретики (отдельные препараты) & $4(11.8)$ \\
\hline 3 & Calcium channel blockers + ARB (prescribed separately) / Антагонисты кальция+БРА (отдельные препараты) & $3(8.8)$ \\
\hline 4 & Beta blockers + ARB (prescribed separately) / Бета-адреноблокаторы+БРА (отдельные препараты) & $2(5.9)$ \\
\hline 5 & Calcium channel blockers + antiadrenergics (prescribed separately) / Антагонисты кальция+антиадренергические средства (отдельные препараты) & $2(5.9)$ \\
\hline 6 & Others / Прочие & $5(14.7)$ \\
\hline \multicolumn{3}{|c|}{ ARB - angiotensin II receptor blockers, ACE - angiotensin converting enzyme } \\
\hline \multicolumn{3}{|c|}{ БРА - блокатор рецепторов ангиотензина II } \\
\hline
\end{tabular}

Table 4. Distribution of the five major antihypertensive drug groups according to the govermental and private hospital physicians

Таблица 4. Распределение пяти основных групп антигипертензивных препаратов в зависимости от статуса лечебного учреждения (государственное или частное)

\begin{tabular}{|c|c|c|c|c|c|}
\hline & $\begin{array}{c}\text { Beta-blockers } \\
\text { Бета-блокаторы } \\
\text { n=36 }\end{array}$ & $\begin{array}{c}\text { ARBs } \\
\text { БPA } \\
n=35\end{array}$ & $\begin{array}{c}\text { Calcium channel blockers } \\
\text { Антагонисты кальция } \\
\text { n=29 }\end{array}$ & $\begin{array}{c}\text { ACE İnhibitors } \\
\text { Ингибиторы АПФ } \\
\text { n=25 }\end{array}$ & $\begin{array}{c}\text { Diuretics } \\
\text { Диуретики } \\
\mathrm{n}=21 \\
\end{array}$ \\
\hline \multicolumn{6}{|l|}{ Governmental hospital physicians, n } \\
\hline Государственное лечебное учреждение, n & 20 & 20 & 13 & 14 & 9 \\
\hline \multicolumn{6}{|l|}{ Private hospital physicians, n } \\
\hline Частное лечебное учреждение, n & 16 & 15 & 12 & 15 & 12 \\
\hline \multicolumn{6}{|l|}{ ARB - angiotensin || receptor blockers } \\
\hline \multicolumn{6}{|c|}{ БРА - блокатор рецепторов ангиотензина II, АПФ - ангиотензин-превращающий фермент } \\
\hline
\end{tabular}

Table 5. Distribution of the five major antihypertensive drug groups according to the gender of the physicians

Таблица 5. Распределение пяти основных групп антигипертензивных препаратов в зависимости от пола лечащего врача

\begin{tabular}{|c|c|c|c|c|c|}
\hline & $\begin{array}{c}\text { Beta-blockers } \\
\text { Бета-адреноблокаторы } \\
\text { n=29 }\end{array}$ & $\begin{array}{c}\text { ARBs } \\
\text { EPA } \\
n=30\end{array}$ & $\begin{array}{c}\text { Calcium channel blockers } \\
\text { Антагонисты кальция } \\
\text { n=29 }\end{array}$ & $\begin{array}{c}\text { ACE İnhibitors } \\
\text { Ингибиторы АПФ } \\
\mathrm{n}=28\end{array}$ & $\begin{array}{c}\text { Diuretics } \\
\text { Диуретики } \\
\mathrm{n}=13\end{array}$ \\
\hline Female, n / Женский, n & 10 & 12 & 5 & 14 & 4 \\
\hline Male, n / Мужской, n & 19 & 18 & 24 & 14 & 9 \\
\hline \multicolumn{6}{|c|}{ ARB - angiotensin II receptor blockers } \\
\hline \multicolumn{6}{|c|}{ БРА - блокатор рецепторов ангиотензина ІІ, АПФ - ангиотензин-превращающий фермент } \\
\hline
\end{tabular}

tion, according to the ATC-3 subclassification the most preferred drug groups were the beta blockers, ARBs and ACE-inhibitors (Table 4).

It was also observed that according to the ATC-2 subclassification both male and female physicians most commonly prescribe the agents acting on renin-angiotensin system (ARBs + ACE inhibitors). However, female physicians mostly prescribed ACE-inhibitors, $A R B s$ and beta blockers whereas male physicians mainly prescribed calcium channel blockers and betablockers. ARBs were prescribed as a second choice drug (Table 5). увеличивать риск неблагоприятного взаимодействия лекарственных веществ, влиять на сопутствующие заболевания, вызывать побочные эффекты [14]. Наше исследование показало, что в назначениях содержится 40\% препаратов, которые не являются антигипертензивными. Одной из причин такого назначения может быть наличие хотя бы одного сопутствующего заболевания. К сожалению, из-за отсутствия полной информации в рецептах невозможно точно установить сопутствующие патологии. С другой стороны, среднее количество антигипертензивных препаратов в рецепте было $1,2 \pm 0,6$, что соотносится с данными турецкого иссле- 


\section{Discussion}

We aimed to analyses the antihypertensive drug prescription patterns of the physicians by evaluating scripts that included antihypertensive agents. Considering the relevant literature, this is the first pharmacoepidemiological study covering the rational use of medicine (RUM) approaches of the physicians in terms of antihypertensive drug prescription in NC. Identifying imperfections in prescribing patterns reveals the requirement of educational interventions to improve the prescribing behavior of the physicians.

In this study, the average number of drugs per prescription (2.1) was similar with the study conducted in Bahrain (2.12) and slightly lower than the study conducted in Istanbul, Turkey (3.8) $[12,13]$. Polypharmacy is a problem for rational drug prescribing, especially, in elderly patients. Polypharmacy is undesirable and might be associated with an increased risk of drug/drug and drug/disease interactions and adverse effects [14]. In this study, it was observed that $40 \%$ of the prescribed drugs were the agents other than the antihypertensive. One reason of this polypharmacy might be caused due to the presence of at least one co-morbidity together with hypertension. Unfortunately, because of the lack of adequate data on the prescriptions, the co-morbidities of those patients could not be evaluated. On the other hand the mean number of the antihypertensive(s) per prescription was $1.2 \pm 0.6$ which was similar with the findings of the study conducted in Turkey (1.2 \pm 0.6 ) and was slightly lower than those findings of the study conducted in Bahrain (1.5 \pm 0.6$)[12,13]$. These findings showed that monotherapy was more common in NC which is crucial for RUM principles.

As defined by WHO, RUM requires patients to use the medication within the appropriate dose and duration according to their clinical needs [8]. For the offering of the right medication to the patient by the pharmacist, the information in prescriptions such as dosage, pharmaceutical form, duration of usage etc. must be correctly specified. In this study it was recognized that the prescriptions had some imperfections in terms of dosage, pharmaceutical form and usage instructions. This finding also points to the need of educational interventions.

For the management of hypertension several international guidelines have been updated [4-7]. According to these guidelines in patients with uncomplicated hypertension, the recommended first-choice medications are diuretics, ACE inhibitors, ARBs and calcium channel blockers [4-7]. Previously, beta-blockers were recommended for initial therapy, but due to the failure of their importance in coronary artery disease caused them to do not recommend as a first-line agent for patients with hypertension not complicated by other disorders [4-7]. дования $(1,2 \pm 0,6)$, и несколько ниже показателей, полученных в Бахрейне $(1,5 \pm 0,6)[12,13]$. Эти данные свидетельствуют о том, что в Северном Кипре монотерапия более распространена, что важно для соблюдения принципов рациональной фармакотерапии.

По определению ВОЗ для рационального использования лекарственных средств требуется, чтобы пациенты получали медикаментозное лечение, соответствующее клиническим показаниям, в таких дозах, которые отвечают их индивидуальным потребностям [8]. Чтобы фармацевт мог предложить пациенту нужное лекарственное средство, в рецепте должна быть корректная информация о режиме дозирования, лекарственной форме, длительности лечения и т.д. Настоящее исследование показало, что рецепты имеют некоторые недочеты касательно режима дозирования, лекарственной формы и инструкций по применению. Эти результаты также указывают на необходимость проведения обучающих мероприятий.

Существуют несколько современных международных сводов рекомендаций для лечения гипертензии [4-7]. Согласно этим клиническим руководствам у пациентов с неосложненной АГ препаратами первого выбора являются диуретики, ингибиторы АПФ, БРА и антагонисты кальция [4-7]. Ранее бета-адреноблокаторы были рекомендованы для начальной терапии, но в силу недоказанности их значимости при ишемической болезни сердца их не рекомендуют в качестве препарата выбора при АГ, не осложненной другими событиями [4-7].

По данным нашего исследования, в противовес клиническим руководствам по подклассу ATX-2, бета-адреноблокаторы являлись вторыми по частоте назначения препаратами, в то время как по подклассу ATX-5 бетаадреноблокатор метопролол был препаратом выбора при лечении АГ. В дополнение к этому, в отличие от рекомендаций по подклассу ATX-2, диуретики являлись четвертыми по частоте назначения; по подклассу ATX-5 петлевой диуретик фуросемид был третьим по назначаемости препаратом при АГ. По данным исследований, проведенных в Турции и Нигерии, диуретики являлись наиболее предпочтительными средствами $[15,16]$. Настоящее исследование выявило, что назначенные диуретики в основном относились к группам петлевых и калийсберегающих, и такие результаты позволили предположить, что врачи избегают применять диуретики в качестве препаратов выбора из-за их побочного действия в виде гипокалиемии.

Полученные данные совпадают с результатами исследований в Германии и Бахрейне, где бета-адреноблокаторы были наиболее часто выбираемыми препаратами $[17,18]$. Однако согласно клиническим руководствам и данным некоторых исследований применять бета-адреноблокаторы не рекомендуется, если нет сопутствующих патологий, таких как ишемическая болезнь сердца или перенесенный инфаркт миокарда [4-7]. В 
In this study, in contrast to guidelines according to the ATC-2 subclassification the beta-blockers were the second most chosen drug whereas according to the ATC-5 subclassification beta-blocker, metoprolol was the first-choice drug in the hypertension treatment. In addition, unlike the guidelines according to the ATC-2 subclassification, diuretics were the fourth chosen drug whereas according to the ATC- 5 subclassification a high-ceiling diuretic, furosemide was the third-chosen drug in hypertension treatment. In a study conducted in Turkey and in Nigeria, diuretics was the most preferred drugs $[15,16]$. In this study, it was observed that the most common prescribed diuretics were the highceiling diuretics and potassium-sparing agents. These findings suggested that physicians might avoid using diuretics as a first-line treatment due to their hypokalemic side-effects.

Similarly, to our findings, it was found that betablockers were the most common chosen medications in studies conducted in Germany and Bahrain $[17,18]$. However, as guidelines and some studies suggest, the usage of beta-blockers were not recommended in the absence of co-morbidity such as coronary artery disease and post myocardial infarction [4-7]. However, in this study because of the lack of the data, the relation of the co-morbidities and the usage of beta-blockers could not been evaluated.

As suggested by the guidelines, the most common prescribed drugs in this study were the agents acting on renin-angiotensin-system. In this group $44.4 \%$ of the drugs belonged to the ACE-inhibitors whereas $55.6 \%$ of them belonged to the angiotensin receptor blockers (ARB). Turkish Hypertension Consensus does not distinguish ACE inhibitors from the ARBs [16]. Therefore, this could be the reason to have a tendency to prescribe an ARB, even though ACE inhibitors are more affordable. Unlike the present study, ARBs were the least chosen drugs in studies conducted in Nigeria and Bahrain $[12,15]$. This may be due to the high cost of the newer ARBs. On the other hand, in the studies conducted in Bosnia and Herzegovina, it was reported that the ACE inhibitors were the most commonly prescribed antihypertensive [19]. In clinical trials, it was reported that both of them have equal effectiveness to reduce blood pressure however they might have different efficacy in terms of some co-morbidities [5,6]. For instance, ACE inhibitors are recommended for hypertensive patients with a history of myocardial infarction and chronic heart failure. ARBs are recommended in case that ACE inhibitors are not tolerated well $[5,6]$. On the other hand, Turkish hypertension consensus report recommends the usage of ACE inhibitors or ARBs for the first line treatment in those patients with diabetes mellitus and avoids using diuretics which is contraindi- настоящем исследовании оценить соотношение сопутствующих патологий и назначения бета-блокаторов не удалось из-за нехватки данных.

В соответствии с клиническими руководствами наиболее часто назначаемыми препаратами в нашем исследовании оказались вещества, влияющие на ренинангиотензиновую систему. В этой группе 44,4\% препаратов относились к ингибиторам АПФ, в то время как 55,6\% являлись блокаторами рецепторов ангиотензина (БРА). Турецкий Консенсус по лечению гипертонии не выделяет ингибиторы АПФ отдельно от БРА [16], следовательно, это может служить причиной частого назначения БРА, хотя ингибиторы АПФ более доступны по цене. В отличие от нашего исследования, БРА были самыми редко назначаемыми препаратами по данным исследований, проведенных в Нигерии и Бахрейне $[12,15]$, это может быть следствием высокой стоимости современных БРА. С другой стороны, исследования, проведенные в Боснии и Герцеговине, показали, что ингибиторы АПФ назначаются наиболее часто в качестве антигипертензивных препаратов [19]. По данным клинических исследований обе группы препаратов имеют равную эффективность в снижении артериального давления, но могут иметь разную эффективность при некоторых сопутствующих заболеваниях $[5,6]$. Например, ингибиторы АПФ рекомендованы для лечения пациентов с АГ, имеющих в анамнезе инфаркт миокарда и хроническую сердечную недостаточность, БРА рекомендованы в случае плохой переносимости ингибиторов АПФ $[5,6]$. В то же время положения Турецкого Консенсуса по гипертензии рекомендуют использование ингибиторов АПФ или БРА в качестве препаратов выбора у пациентов с сахарным диабетом и избегают использования диуретиков, которые противопоказаны [16]. Кроме того, в одном исследовании был заявлен ренопротективный эффект регулярного лечения ингибиторами АПФ или БРА [20]. Некоторые клинические руководства и исследования предполагают, что применение антагонистов кальция и диуретиков в дополнение к ингибиторам АПФ и БРА эффективно снижает АД у пациентов с диабетом $[5,6,20]$. Одним из самых значимых ограничений настоящего исследования является невозможность оценки рационального использования антигипертензивных препаратов у пациентов с диабетом, так как отсутствуют данные о сопутствующих патологиях.

Согласно клиническим руководствам, комбинированная терапия, определяемая как использование минимум двух антигипертензивных препаратов, должна рассматриваться в случаях очень высокого базового уровня артериального давления или при высоком сердечно-сосудистом риске $[5,6]$. В нашем исследовании наиболее частой комбинацией является сочетание БРА и диуретика в форме комбинированного препарата, что согласуется с результатами исследования G. Yürüyen и 
cated [16]. In addition, in a study the renoprotective role of regular ACE inhibitor or ARB treatment was stated [20]. However, some guidelines and studies suggested that the usage of the calcium channel blockers and diuretics in addition to ACE inhibitors and ARBs effectively lower blood pressure in diabetic patients $[5,6,20]$. In present study, it was one of the most significant limitations that the rational use of antihypertensive in diabetic patients could not be evaluated due to lack of the co-morbidity data.

According to the guidelines, combination therapy, defined by the use of at least two antihypertensive drugs, should be considered for the patients with very high baseline blood pressure or at high cardiovascular risk $[5,6]$. In this study, the most commonly used combination was an ARB and a diuretic in a form of fixed combination which was similar with those findings reported by G. Yürüyen et al. [16]. The usage of a combination pill with fixed dose formulations was originally proposed to overcome two problems: the difficulty of compliance to treatment regarding multiple drugs; and the inadequate dosages prescribed during clinical practice [21]. According to the NICE guideline, if the blood pressure were uncontrolled by a monotherapy, unlike the present study, an ACE inhibitor or ARB and a calcium channel blocker combination is recommended. If calcium channel blocker is not suitable due to oedema and intolerance and the risk of heart failure, a thiazidelike diuretic should be chosen [7]. Similarly, some studies also suggested that ACE and calcium channel blockers is superior to the combination of ACE inhibitors and diuretics and beta-blockers and diuretics $[22,23]$. In addition, a systemic review suggested the equal effectiveness of all drug combinations in hypertensive patients without co-morbidities, but differs in their efficacy in preventing certain outcomes [24]. It was shown that calcium channel blockers reduced mortality and the incidence of stroke when compared to beta-blockers whereas ACE inhibitors were reported to reduce the incidence of myocardial infarction when compared to beta blockers [24]. In addition a guideline stated the usage of ACE inhibitor or ARB + calcium channel blockers for antihypertensive patients with diabetes and/or lipid abnormalities, ACE inhibitor or ARB + thiazide diuretics for antihypertensive patients with heart failure or post stroke, ACE inhibitor or ARB + beta-blockers for antihypertensive patients with post myocardial infarction or heart failure, beta-blocker + dihydropyridine calcium channel blocker for antihypertensive patients with symptomatic coronary heart disease [5]. It is thought that the combining of two classes of antihypertensive drug lowers blood pressure more than doubling the dosage of one drug. As suggested by guidelines, if baseline blood pressure is very high, treatment соавт. [16]. Использование комбинированного препарата с фиксированной дозировкой изначально должно было исключить две проблемы: низкую приверженность лечению из-за применения нескольких препаратов и нерациональную схему приема лекарств, назначаемых в клинической практике [21]. По рекомендациям NICE (Национальный институт здравоохранения и совершенства медицинской помощи Великобритании), если контроль АД не достигается при монотерапии, то рекомендуется комбинация ингибитора АПФ или БРА с антагонистом кальция, что отличается от результатов настоящего исследования. Если антагонист кальция не может применяться из-за отечности и непереносимости, а также риска сердечной недостаточности, то следует выбирать тиазидный диуретик [7]. Аналогично некоторые исследования предполагают, что БРА и антагонисты кальция имеют превосходство по эффективности над комбинациями ингибиторов АПФ и диуретиков, бета-адреноблокаторов и диуретиков $[22,23]$. Кроме того, в одном исследовании рассматривается равная эффективность всех комбинаций препаратов в лечении пациентов с неосложненной АГ, но выявляются отличия их эффективности в профилактике определенных исходов [24]. Было доказано, что по сравнению с бета-адреноблокаторами антагонисты кальция снижают уровень смертности и частоту возникновения инсульта, тогда как ингибиторы АПФ уменьшают риск развития инфаркта миокарда [24]. Дополнительно в клиническом руководстве рекомендовано применение для антигипертензивной терапии ингибитора АПФ или БРА и антагониста кальция у пациентов с диабетом и/или нарушением липидного обмена; ингибитора АПФ или БРА с тиазидным диуретиком у пациентов с сердечной недостаточностью или перенесенным инсультом; ингибитора АПФ или БРА с бетаадреноблокаторами у пациентов с перенесенным инфарктом миокарда или сердечной недостаточностью; бета-адреноблокатора в сочетании с дигидропиридиновым антагонистом кальция у пациентов с ишемической болезнью сердца и клинической симптоматикой [5]. Считается, что применение двух антигипертензивных средств разных классов понижает АД более эффективно, чем увеличенная вдвое дозировка одного из них. Как предлагают клинические руководства, при высоком базовом уровне АД более предпочтительна комбинированная начальная терапия (два препарата), чем монотерапия (одним лекарственным средством) [5,6]. Однако следует тщательно оценить полезный эффект и возможные ограничения при назначении комбинированного лечения. Основные преимущества комбинированной терапии - это более быстрое снижение АД и меньшие вариации препаратов и схем назначения. С другой стороны, возможны трудности при оценке эффективности отдельного лекарственного средства при установлении связи нежелательных явлений с конкретным действую- 
might be started with two drug (combination) rather than one (monotherapy) $[5,6]$. However, the benefits and limitations of the combination treatment should be considered carefully. The main benefits of the combination treatment include a more rapid reduction in blood pressure and provide less drug and dosage changes. On the other hand, it is possible to encounter difficulties such as assessment of efficacy of individual drug, attribution of adverse effects to individual drug and single pill combination drugs may not be supported for initiation of antihypertensive therapy [5]. In this study, it was shown that the most chosen combination treatment ( $A R B+$ diuretics) is different from those suggested by guidelines. On the other hand, because the lack of the co-morbidities, the reason of combination treatment is not evaluated completely. This issue is one of the limitations in this study and shows the necessity of educational interventions.

\section{Conclusion}

This study reported that physicians serving in NC performed irrational behaviors in terms of antihypertensive drug choice, writing a legible prescription which contains full information and includes the co-morbidities in order to prescribe the most suitable antihypertensive for the patients as suggested by guidelines. These results indicate the necessity of educational interventions for physicians to disseminate the RUM in NC.

Disclosures. All authors have not disclosed potential conflicts of interest regarding the content of this paper.

\section{References / Литература}

1. Vasan R.S., Beiser A., Seshadri S., et al. Residual lifetime risk for developing hypertension in middleaged women and Men. JAMA. 2002;287:1003-10. DOI:10.1001/jama.287.8.1003.

2. Benowitz N.L. Antihypertensive agents. In: Katzung B.G., Masters S.B., Trevor A.J., eds. Basic and Clinical Pharmacology, 12th ed. New York: McGraw Hill; 2012. pp.169-90.

3. Kannel W.B., Gordon T. Evaluation of cardiovascular risk in the elderly: the Framington study. Bulletin of the New York Academy of Medicine. 1978;54(6):573-91.

4. 2013 ESH/ESC guidelines for the management of arterial hypertension: the Task Force for the Management of Arterial Hypertension of the European Society of Hypertension (ESH) and of the European Society of Cardiology (ESC). Eur Heart J. 2013;34(28):2159-219. DOI:10.1093/eurheartj/eht151.

5. Guideline for the diagnosis and management of hypertension in adults 2016. National heart foundation of Australia [cited by Jul 02, 2019]. Available from: https://www.heartfoundation.org.au/images/uploads/publications/PRO-167 Hypertension-guideline-2016 WEB.pdf.

6. 2017 ACC/AHA/AAPA/ABC/ACPM/ĀAGS/APhA/ASH/ASPC/NMA/PCNA Guideline for the Prevention, Detection, Evaluation, and Management of High Blood Pressure in Adults: Executive Summary: A Report of the American College of Cardiology/American Heart Association Task Force on Clinical Practice Guidelines. Hypertension. 2018;71(6):1269-324. DOI:10.1161/HYP. 000000000000066

7. NICE guideline. Hypertension in adults: diagnosis and management [cited by May 28, 2019]. Available from: https://www.nice.org.uk/guidance/cg127/chapter/1-Guidance\#initiating-and-monitoring-antihypertensive-drug-treatment-including-blood-pressure-targets-2.

8. WHO. The rational use of drugs: report of the conference of experts. Nairobi: World Health Organization; 1985.

9. Rizzo J.A., Simons W.R. Variations in compliance among hypertensive patients by drug class: implications for health care costs. Clin Ther. 1997;19(6):1446-57. DOl:10.1016/501492918(97)80018-5

10. Fulhaber H.D., Luft F.C. Treatment of high blood pressurein Germany. Am J Hypertens. 1998:11:7503. DOI:10.1016/s0895-7061(98)00068-5 щим веществом. Кроме того, фиксированные комбинации могут не подходить для начальной антигипертензивной терапии [5]. В настоящем исследовании отмечено, что наиболее частая комбинация (БРА + диуретики) не отражает действующие клинические рекомендации. Стоит отметить, что из-за отсутствия данных о сопутствующих заболеваниях достоверно оценить назначение комбинированной терапии не удалось. Это является одним из ограничений настоящего исследования, и еще раз доказывает необходимость обучающих мероприятий.

\section{Заключение}

Проведенное исследование показало, что врачи Северного Кипра не всегда рационально назначают антигипертензивные препараты, рецепты не всегда содержат полную информацию, в том числе, о сопутствующих заболеваниях, что важно для выбора наиболее адекватной антигипертензивной терапии согласно клиническим руководствам. Полученные результаты свидетельствуют о необходимости проведения обучения для врачей с целью распространения принципов рационального использования лекарственных средств на Северном Кипре.

Конфликт интересов. Все авторы заявляют об отсутствии потенциального конфликта интересов, требующего раскрытия в данной статье.

11. State Planning Organization. Population and indicators [cited by Jul 02, 2019]. Available from: http://www.devplan.org/nufus-2011/nufus\%20ikinci_.pdf.

12. Jassim Al Khaja K.A., Sequeira R.P., Mathur V.S. Rational Pharmacotherapy of hypertension in the elderly: analysis of the choice and dosage drugs. J Clin Phar Therap. 2001;26:33-42. DOI:10.1111/j.1365-2710.2001.00324.x

13. Akici A., Kalaça S., Uğurlu U., Toklu H.Z., Oktay ş. Antihypertensive drug utilization at health centers in a district of Istanbul. Pharm World Sci. 2007;29:116-21. DOI:10.1007/s11096-007-9103-5.

14. Obreli Neto P.R., Nobili A., de Lyra D.P. Jr, et al. Incidence and predictors of adverse drug reactions caused by drug-drug interactions in elderly outpatients. A prospective cohort study. J Pharm Pharm Sci. 2012;15(2):332-43

15. Bakare O.Q., Akinyinka M.R., Goodman O., et al. Antihypertensive use, prescription patterns and cost of medications in a Teaching Hospital in Lagos, Nigeria. Niger I Clin Pract. 2016;19:668-72. DOI:10.4103/1119-3077.188709

16. Yürüyen G., Toprak I.D., Toprak Z., et al. Choice of treatment based on Turkish hypertension consensus repost: Do we fellow the recommendations? Turk Kardiyol Dern Ars. 2018;46(1):25-31. DOI:10.5543/tkda.2017.86344

17. Sequeira R.P., Al Khaja K.A., Damanhori A.H.H., Mathur V.S. Prescribing pattern on antihypertensive drugs by family physicians and general practitioners in the primary care setting in Bahrain. J Eval Clin Pract. 2002:8(4):407-14. DOI:10.1046/j.1365-2753.2002.00370.x.

18. Gasse C., Stieber J., Döring A., Keil U., Hense H.W. Population trends in antihypertensive drug use: results from the MONICA Augsburg Project 1984 to 1995. J Clin Epidemiol. 1999;144:726-32. DOI:10.1016/S0895-4356(99)00035-9.

19. Selmanovic K., Zec S.L., Vanis N., et al. Utilisation analysis of antihypertensive drugs in Bosna and Herzegovina for the time-period 2013-2015. Mater Sociomed. 2016;28:116-20.

20. Zanchetti A., Ruilope L.M. Antihypertensive treatment in patients with type-2 diabetes mellitus: what guidance from recent controlled randomized trials? J Hypertens. 2002;20:2099-110. 
21. Secondary prevention of non-communicable diseases in low- and middle-income countries through community-based and health service interventions. Report of the Cambridge Meeting. World Health Organization and Wellcome Trust, 2001 [cited by Jul 02, 2019]. Available from: https://apps.who.int/iris/handle/10665/42567.

22. Jamerson K., Weber M.A., Bakris G.L., et al. Benazepril plus amlodipine or hydrochlorothiazide for hypertension in high-risk patients. N Engl J Med. 2008;359(23):2417-28.

\section{About the Authors:}

Mevhibe Tamirci - PhD (pharmacology), Department

of Pharmacology, Faculty of Pharmacy, European University of Lefke

Rümeysa Demirdamar - PhD (pharmacology), Professor,

Department of Pharmacology, Faculty of Pharmacy, European

University of Lefke.
23. Dahlöf B., Sever P.S., Poulter N.R., et al. Prevention of cardiovascular events with an antihypertensive regimen of amlodipine adding perindopril as required versus atenolol adding bendroflumethiazide as required, in the Anglo-Scandinavian Cardiac Outcomes Trial-Blood Pressure Lowering Arm (ASCOT-BPLA): a multicentre randomised controlled trial. Lancet. 2005;366(9489):895-906. DOI:10.1016/S0140-6736(05)67185-1.

24. Hutton B., Yazdi F., Hersi M., et al. Drug safety and effectiveness network report: A systemic review with network meta-analyses and economic evaluation comparing therapies for hypertension in nondiabetic patients. Ottawa: University of Ottowa, 2014 [cited by Jul 02, 2019]. Available from: www.ruor.uottowa.ca/handle/10393/32292.

\section{Сведения об авторах}

Мевхибе тамирджи - PhD (фармакология),

отдел фармакологии, фармацевтический факультет, Европейский университет Лефке

Рюмейса Демирдамар - PhD (фармакология), профессор, отдел фармакологии, фармацевтический факультет, Европейский университет Лефке 